\title{
DATOS SOBRE EL LEÍSMO Y LAÍSMO DE PERSONA EN EL HABLA DE LA CIUDAD DE BURGOS
}

\author{
F. Miguel Martínez Martín \\ U.N.E.D.
}

\section{INTRODUCCIÓN}

1.1. En este trabajo vamos a dar algunos datos sobre la situación, en la ciudad de Burgos ', del leísmo y laísmo de persona, fenómenos intimamente relacionados, pero cuyas características estructurales no están muy claras ${ }^{2}$. Su difusión tampoco es muy conocida

1 Los datos de este estudio proceden de una encuesta hecha en 1978 a cincuenta informantes de origen burgalés. Los distintos grupos de hablantes han sido seleccionados por cuotas mediante el procedimiento de *cadenax, siendo construidos sus primeros eslabones con métodos de muestreo aleatorio. Nuestra muestra es adecuada, representativa y suficiente en número, pues contamos con grupos de hablantes heterogéneos en correspondencia con las tendencias generales de la base de muestra, que, en nuestro caso, es el Censo de 1970 y el Padrón Municipal de 1975. Estas cuotas generan una división por sexo, por tres grupos de edad (de los veinte a los treinta y cinco años, de los treinta y cinco a los cincuenta, y de los cincuenta en adelante) y por tres niveles socioculturales, centrados, sobre todo, en el grado de instrucción (superior, medio, inferior).

Para mayor información sobre la extracción de la muestra y para otras consideraciones metodológicas pueden verse los trabajos: MarTfNez MARTfN, F. Miguel: Fonética y sociolingüística en la ciudad de Burgos, Madrid, C. S. I. C., 1983, y « La sustitución de cantara (cantase) por cantaria en el habla de la ciudad de Burgos*, en Lingüistica española actual, V, 1983, págs. 179-204.

2 Véanse, por ejemplo, los trabajos de LAPESA, Rafael: «Sobre los orígenes y evolución del leísmo, laísmo y loísmo», en Festchrift W. v. Wartburg, I, Tübingen, Max Niemayer, 1968, págs. 523-551. GaRcfa, Erica G.: The Role of Theory in Linguistic Analysis: The Spanish Pronoum System, North-Holland, Linguistic Series, 1975. Marcos Marin, F.: Estudios sobre el pronombre, Madrid, Gredos, 1978. Garcfa GonzALEZ, F.: «El leísmo en Santander», en Estudios ofrecidos a Emilio Alarcos Llorach, III, Oviedo, 1978, págs. 87-101. KLEIN-ANDREU, Flora: «Distintos sistemas de empleo de 'le', 'la', 'lo'. Perspectiva sincrónica, diacró- 
empíricamente aunque se intuye una *fuerza de difusión avasalladora», tanto social como geográfica, que parece partir de Castilla. Sin embargo, el mismo autor que esto dice califica el laísmo como "vulgarismo flagrante y, por ello, totalmente inadmisible" y el leísmo de persona como "no verdaderamente correcto ni verdaderamente elegante" ${ }^{3}$.

En las líneas que siguen no pretendemos dar doctrina, sino más bien averiguar los rasgos sociolingüísticos que caracterizan a ambas variantes y demostrar, por un lado, la enorme extensión del leísmo y, por otro, la existencia de un polimorfismo alternante con predominio laísta.

La Real Academia se inclina unas veces a favor de un etimologismo estricto y otras veces cede al imperio del uso, como lo prueba la aceptación del laísmo en las primeras ediciones y la del leísmo singular desde la edición de 1854, aunque desde la de 1920 el leísmo singular se tolere de mala gana ${ }^{4}$. Es lógico que la postura académica de inseguridad, criticada por Bello como un adefecto grave de nuestra lengua" 5 , repercuta en los hablantes y manifiesten ellos mismos esta inseguridad.

1.2. El trabajo de Lapesa (1968), que ofrece las líneas maestras para trazar una explicación satisfactoria tanto diacrónica como sincrónica, ha abierto camino a varias investigaciones sobre el tema (véanse, por ejemplo, los títulos de la nota 2). Parece, sin embargo, que encontrar una sistematización simple y exhaustiva de los pronombres personales $l e$, $l a$, lo para cualquier tipo de referente no es tan fácil. Da la impresión de que nos encontramos en una situación parecida a la que denunciaba $R$. J. Cuervo en 1895 cuando dice que "no se descubre camino por donde pueda remediarse» ${ }^{6}$. Por el momento, y si nos ceñimos al rasgo (+ persona), se puede afirmar que existen dos sistemas cuya diferencia no es de "sentido", sino de difusión geográfica y social ?:

nica y sociolingüístican, en Thesaurus Boletin Instituto Caro y Cuervo, XXXVI, Bogotá, 1981, págs. 284-304. MoNGE, Félix: "Notas a una hipótesis sobre el leísmo», en Serta Philologica F. Lázaro Carreter, I, Madrid, Cátedra, 1983, págs. 441-455.

3 LloRente Maldonado, Antonio: "Consideraciones sobre el español actual», en Anuario de Letras, XVIII, México, 1980, págs. 5-61 (citas de las págs. 26 y 24).

4 LAPESA, Rafael, op. cit. (1968), pág. 546, Gramática de la lengua española, Real Academia Española, Madrid, Perlado Sáez y Compañia, 1924, y Esbozo de una nueva gramática de la lengua española, Madrid, Espasa-Calpe, 1973.

5 Bello, A., y Cuervo, R. J.: Gramática de la lengua castellana, Buenos Aires, Sopena, 1964, 7.: edición, pág. 304, n. 121.

6 Cuervo, R. José: aLos casos enclíticos y proclíticos del pronombre de tercera persona en castellano», en Romania, XXIV, 1985, págs. 95-113 y 217-263) (cita de la pág. 244).

7 Monge, Félix, op. cit., pág. 449. 
- El sistema etimológico o funcional, de todos conocido, es el que se mantiene con mayor rigor como norma lingüística culta y es el más extendido entre andaluces, murcianos, aragoneses, hispanoamericanos e hispanohablantes de Cataluña, Levante y Baleares ${ }^{8}$. Está atacado por el leísmo de persona, pues se tolera, en parte, el uso de le en singular por $l o$.

- El sistema de distinción por el género del referente busca una organización sustentada por la coherencia y sobre la fuerza analógica que el morfema género presenta. Este sistema muestra desequilibrios provocados por el rechazo académico o por otras fuerzas: hay alternancia $l e(s)-l a(s)$ en objeto indirecto femenino y presencia de algunos casos de $l e(s)$ por $l a(s)$ en objeto directo femenino?.

Según opinión general ${ }^{10}$, el sistema basado en la distinción por el género y no por la función prendió en Castilla desde muy temprano formando paradigma con éste-ésta-esto y con aquél-aquéllaaquello. Pero a pesar de la seguridad analógica que proporciona el paradigma, parece que no se ha logrado eliminar el sistema funcional dentro de las dos Castillas, foco principal de expansión de la isoglosa. Dentro de la isoglosa se encuentra la ciudad de Burgos, representante, por derecho propio, de la prestigiosa modalidad castellana noroccidental. Esta modalidad ha sido el "norte» del "vasto complejo dialectal» castellano, por lo menos hasta el siglo XIX. En estos momentos es Madrid, "enclave de pronunciación norteña», la ciudad que parece detentar el modelo idiomático ${ }^{11}$.

8 Llorente, Antonio, op. cit., pág. 26.

9 Este último extremo permite al profesor Marcos Marín proponer un tercer sistema en germen, el sistema leísta. El autor explica la presencia de este sistema por la existencia de una función objeto de referencia pasiva sin interés (- persona) y de otra función objeto de interés personal o de protagonismo (+ persona), en la que cabe, junto a los legítimos objetos directo e indirecto masculinos, gran parte de los objetos directos personales femeninos realizados con le (-s).

Tanto Cuervo, R. J., op. cit., pág. 112, como LaPESA, R., op. cit. (1968), pág. 541, califican la utilización de le por la objeto directo femenino como algo rarísimo -referido a personas-. y piensan que sería mejor interpretarlo como un tipo de objeto indirecto, como la formación de una kesfera personal con dativo*.

10 Véanse, por ejemplo, FERNÁndez RamfRez, Salvador: Gramática española, Madrid, Manuales de la Revista de Occidente, 1951, pág. 202, y LAPESA, R., op. cit. (1968), págs. 523 y 541 .

11 LAPESA, R., op. cit. (1968), pág. 551; LAPESA, R.: Historia de la lengua española, Madrid, Gredos, 1980, 8." edición, pág. 372; LoPE Blanch, J. M.: «El concepto de prestigio y la norma lingüística del español», en Anuario de Letras, X, México, 1972, págs. 29-46 (cita de las págs. 34-36). En fechas próximas aparecerá el trabajo de Quilis, A.; Cantarero, M.; Albala, M. J., y Guerra, R.: Los pro- 


\section{LOS «TESTS» DE ACTITUD}

2.0. Entendemos por actitud una toma de postura ante cualquier variante linguiística, que será positiva (aceptación) o negativa (rechazo) y que está provocada por creencias ${ }^{12}$.

La actitud de los hablantes burgaleses en torno a las variantes objeto directo masculino e indirecto femenino de las formas pronominales átonas de tercera persona, se puede medir mediante la utilización de dos atests»: el «test" de creencia de uso o de autovaloración (qué variante cree el informante que dice generalmente) y el "test" de corrección (qué variante le parece la más correcta). La comparación de ambas medidas entre sí y con los datos de uso real en el estilo más formal ${ }^{13}$ nos dará datos sobre la inseguridad lingüística, típica situación de lucha de sistemas, y que el hablante burgalés manifiesta al caracterizar su habla como incorrecta.

El problema de la inseguridad del laísmo y leísmo ha preocupado, desde hace tiempo, a algunos investigadores. Así, Salvá, en su Gramática, dice que lo general es la "incertidumbre», y A. Bello acusa a esta incertidumbre de ser un "grave defecto». Y siempre se llega a la conclusión de la existencia de una lucha de sistemas que nadie sabe en qué va a acabar, a pesar de los esfuerzos de la Real Academia por dar normas que regulen su uso ${ }^{14}$.

\subsection{Le $y$ la, objeto indirecto femenino}

Veremos, en primer lugar, la valoración de las variantes del objeto indirecto femenino.

nombres átonos la, lo, le en el habla de Madrid: Estudio descriptivo, Madrid, C.S. I. C.

12 LOPEZ MORALES, H.: «Sociolingüísticax, en varios: Lengua española, II (para Filosofía y Ciencias de la Educación). Madrid, U. N. E.E.D., 1978, pág. XXV/3.

13 En nuestra investigación consideramos tres registros o estilos contextuales. En el estilo contextual slecturax, el investigador extrega al informante unas fichas con frases que tiene que completar; el grado de consciencia linguíistica es, necesariamente, muy elevado. Las fichas son del siguiente tipo: Di una manzana a Luisa. Complete esta frase: «A Luisa... di una manzana.. El estilo «respuesta* presenta el mismo procedimiento, pero el informante no tiene fichas, sino que oye la pregunta del investigador intercalada con otras sobre cuestiones distintas; el grado de consciencia sobre el fenómeno que investigamos es menor que en el estilo «lectura». En el estilo contextual "conversación»-una verdadera conversación informal de unos veinte minutos-, el grado de consciencia ante las variantes linguísticas es de suponer que sea muy bajo.

14 Véanse SalvA, Vicente: Gramática de la lengua castellana, Paris, Garnier Hermanos, 1883, 10.' edición, pág. 153; Bello, A., y Cuervo, R. J., op. cit. (1964), pág. 304, y Cukrvo, R. J., op. cit. (1895), pág. 233. 
En el experimento usamos la siguiente pregunta para ser contestada por los 50 informantes:

- «Compré una muñeca a Luisa.» Cuál dice usted y cuál le parece la más correcta de estas dos frases: aLa compré una muñeca" o "Le compré una muñeca" (se sobrentiende a Luisa).

En los datos del cuadro 1 el predominio de le irá marcado con el signo + y el de la con el signo - En el gráfico el signo + ocupa el cuadrante por encima de la línea de abscisas y el signo - ocupará el cuadrante por debajo de la misma línea.

2.1.1. "Test" de creencia de uso. De los 50 informantes encuestados hay un 22 por 100 más (12 en números absolutos) que piensan que cuando hablan dicen $l a$; los restantes, como es lógico, se distribuyeron en partes iguales entre la y le. El predominio, aunque corto, es a favor de la variante $l a$, del laísmo; conclusión de acorde con la caracterización final a la que llegamos en este trabajo.

- Distribución por generaciones: En la primera y segunda generación hay, respectivamente, un 35 por 100 y un 45 por 100 más de informantes que opinan que dicen la, mientras que la tercera generación muestra un 23 por 100 más de informantes que creen que dicen $l e$. Parece que puede existir un progreso del laismo observable entre generaciones, como señala Llorente Maldonado en su artículo citado en la nota 2.

- Distribución por niveles socioculturales: El nivel superior ofrece un predominio muy corto de informantes que creen que dicen le, sólo un 17 por $100 ; 0$, lo que es lo mismo, sólo hay dos informantes más que piensan que dicen le. De los diez restantes que componen el nivel sociocultural superior la mitad se inclina hacia la y la otra mitad hacia le. Los niveles medio e inferior muestran un predominio de seis y siete informantes más, respectivamente, $o$ un 33 por 100 y un 35 por 100 más de los que creen que dicen la.

La preferencia por la aumenta conforme vamos descendiendo en la escala sociocultural, pero el grupo medio imita al inferior, no al superior, por lo que no parece que el uso de le por la objeto indirecto femenino muestre un gran prestigio, hecho que veremos cumplirse en los datos reales.

- En la distribución por sexos predomina, en ambos, la creencia de uso de la -el indice es siempre negativo-, pero este predominio es mayor en las mujeres que en los varones, 


\section{CUADRO Y GRAFICO 1}

Indices de creencia de uso, de corrección y uso real para las formas le, la del objeto indirecto femenino, por variables sociales; siendo $n_{1}=$ informantes que dicen le $y n_{2}=$ informantes que dicen la. El

$$
\text { indice }=\frac{n_{1}-n_{2}}{N}
$$

\begin{tabular}{|c|c|c|c|}
\hline & $\begin{array}{l}\text { Indice de } \\
\text { creencia } \\
\text { de uso }\end{array}$ & $\begin{array}{l}\text { Indice de } \\
\text { corrección }\end{array}$ & $\begin{array}{l}\text { Indice de } \\
\text { uso real (es- } \\
\text { tilo formal) }\end{array}$ \\
\hline \multicolumn{4}{|l|}{ Generaciones: } \\
\hline $\begin{array}{lllllllll}\operatorname{Primera} & \ldots & \ldots & \ldots & \ldots & \ldots & \ldots & \ldots & \ldots \\
\operatorname{Segunda}^{\operatorname{Seg}} & \ldots & \ldots & \ldots & \ldots & \ldots & \ldots & \ldots & \ldots \\
\operatorname{Tercera} & \ldots & \ldots & \ldots & \ldots & \ldots & \ldots & \ldots & \ldots\end{array}$ & $\begin{array}{r}-0,35 \\
-0,41 \\
+0,23\end{array}$ & $\begin{array}{l}+0,35 \\
+0,06 \\
+0,23\end{array}$ & $\begin{array}{r}+0,17 \\
-0,22 \\
+0,08\end{array}$ \\
\hline \multicolumn{4}{|l|}{ Niveles socioculturales: } \\
\hline 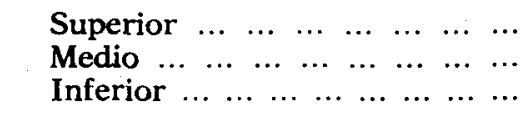 & $\begin{array}{r}+0,17 \\
-0,33 \\
-0,35\end{array}$ & $\begin{array}{l}+0,67 \\
+0,11 \\
+0,05\end{array}$ & $\begin{array}{r}+0,50 \\
-0,04 \\
-0,23\end{array}$ \\
\hline \multicolumn{4}{|l|}{ Grupos de sexo: } \\
\hline $\begin{array}{lllllllll}\text { Varones } & \ldots & \ldots & \ldots & \ldots & \ldots & \ldots & \ldots & \ldots \\
\text { Mujeres } & \ldots & \ldots & \ldots & \ldots & \ldots & \ldots & \ldots & \ldots\end{array}$ & $\begin{array}{l}-0,13 \\
-0,31\end{array}$ & $\begin{array}{l}+0,21 \\
+0,23\end{array}$ & $\begin{array}{r}-0,03 \\
+0,05\end{array}$ \\
\hline Totales $\ldots \ldots \ldots$ & $-0,22$ & $+0,22$ & $+0,01$ \\
\hline
\end{tabular}

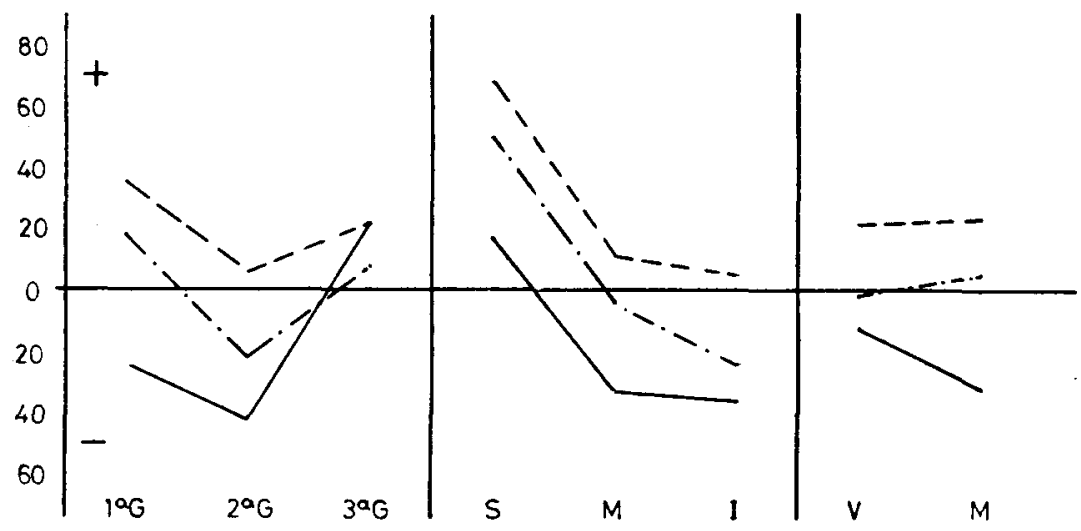

creencia - ; corrección - - - -; uso real -. -... 
lo que vuelve a confirmar la hipótesis de que le objeto indirecto femenino no ofrece prestigio suficiente para que las mujeres estén prontas a imitarlo. Lo mismo sucede en los datos reales.

2.1.2. "Test» de corrección. El dato para el total de la población es de signo contrario al aparecido en el "test» de creencia de uso: hay un 22 por 100 más de informantes que piensan que le es el más correcto. El desequilibrio entre ambos "tests» permite lanzar la hipótesis de inseguridad lingiiística.

- Por generaciones, la mayor inseguridad la presenta la primera generación con un 35 por 100 más de informantes que piensa que le es más correcto, cuando el 35 por 100 más de informantes dice que usa la. Sigue la segunda generación con un 6 por 100 más de informantes que opinan que le es más correcto. La tercera generación presenta mayor seguridad, pues mantiene el mismo índice de la en ambos "tests».

- Por niveles socioculturales la inseguridad se manifiesta por igual en todos los grupos, como puede verse por el similar perfil de las curvas que aparece en el gráfico 1.

- Por sexos, aunque la inseguridad es patente en ambos grupos, son las mujeres las que mayor índice de inseguridad presentan.

Al comparar los datos con el índice de uso real en el estilo contextual más formal, el de «Lectura», observamos que el perfil de las curvas de uso real se asemeja más al de corrección que al de creencia de uso o autovaloración y se presenta a una altura superior a la de este último: en Burgos dicen realmente más veces le -cuando la consciencia lingüística es alta- de lo que piensan que dicen, lo que legitima el planteamiento de inseguridad linguística que hemos defendido para el uso de le o la como objeto indirecto femenino, y la seguridad de que estamos ante una variable sociolingüística.

\subsection{Le $y$ lo objeto directo masculino}

La pregunta utilizada fue:

- «Vi a Luis en la calle.» Cuál dice usted y cuál le parece la más correcta de estas dos frases: «Lo vi en la calle» o "Le vi en la calle» (se sobrentiende a Luis).

2.2.1. "Test" de creencia de uso. Podemos observar que la gran mayoria de los informantes opinan que dicen $l e$. Al aislar las varia- 
bles sociales el índice desciende un poco para la segunda generación, para los varones y para el nivel sociocultural inferior, pero, en general, hay pocas diferencias en las variables sociales (ver cuadro 2 y gráfico 2).

2.2.2. "Test" de corrección. El "test» da índices positivos altos, es decir, hay más presencia de le que de lo. Pero el índice general baja mucho al compararlo con el de creencia de uso: así, si para el total de la población había un 76 por 100 de informantes que en el «test» de creencia de uso decían que usaban le (38 más sobre 50), ahora sólo un 36 por 100 más de informantes (18 más sobre 50 ) piensan que $l e$ es más correcto que $l o$. Sigue habiendo inseguridad aunque la línea no sobrepase la zona negativa hacia lo (ver gráfico 2). Al aislar las variables sociales quienes mayor inseguridad presentan son los informantes de la primera y segunda generaciones, los del grupo sociocultural superior y las mujeres; estos grupos son los que dieron un mayor índice de creencia de uso para le.

Si comparamos ambos «tests» con los datos de uso real en el estilo contextual formal -donde suponemos que el informante in. tenta darnos conscientemente su sistema- observamos que el perfil de la curva de uso real se sitúa por encima de ambos perfiles, pero más cerca del de creencia de uso que del de corrección; al contrario de lo que sucedía con la alternancia $l e(l a)$ objeto indirecto femenino.

El hablante burgalés sigue usando le aunque dude en algunos momentos de su corrección; lo acepta como un castellanismo generalizado. Esto no sucede con la alternancia le (la) objeto indirecto femenino, donde el grupo sociocultural superior nunca sobrepasa el límite cero hacia la zona negativa o zona de predominio de la, como veíamos en el gráfico 1.

\section{3. ¿HAY DIFERENCIAS ENTRE SINGULAR Y PLURAL?}

La única concesión que el sistema etimológico normativo concede a los hablantes que tienen en mayor o menor grado un sistema basado en la distinción por el género es el leísmo de persona singular. El leísmo plural no es admitido porque en romance no existió nunca neutro plural, por lo que la ambigüedad era imposible. Incluso suele defenderse que el leísmo plural aparece muy pocas veces 


\section{CUADRO Y GRAFICO 2}

Indices de creencia de uso, de corrección y uso real para las formas le $y$ lo del objeto directo masculino, por variables sociales; siendo $n_{1}=$ informantes que dicen le $y n_{2}=$ informantes que dicen lo. El

$$
\text { indice }=\frac{n_{1}-n_{2}}{N}
$$

\begin{tabular}{|c|c|c|c|}
\hline & $\begin{array}{l}\text { Indice de } \\
\text { creencia } \\
\text { de uso }\end{array}$ & $\begin{array}{l}\text { Indice de } \\
\text { corrección }\end{array}$ & $\begin{array}{l}\text { Indice de } \\
\text { uso real (es- } \\
\text { tilo formal) }\end{array}$ \\
\hline \multicolumn{4}{|l|}{ Generaciones: } \\
\hline $\begin{array}{lllllllll}\operatorname{Primera} & \ldots & \ldots & \ldots & \ldots & \ldots & \ldots & \ldots & \ldots \\
\operatorname{Prg} & & & & & & & & \\
\text { Segunda } & \ldots & \ldots & \ldots & \ldots & \ldots & \ldots & \ldots & \ldots \\
\text { Tercera } & \ldots & \ldots & \ldots & \ldots & \ldots & \ldots & \ldots & \ldots\end{array}$ & $\begin{array}{l}+0,80 \\
+0,65 \\
+0,85\end{array}$ & $\begin{array}{l}+0,30 \\
+0,18 \\
+0,69\end{array}$ & $\begin{array}{l}+0,90 \\
+0,97 \\
+0,92\end{array}$ \\
\hline \multicolumn{4}{|l|}{ Niveles socioculturales: } \\
\hline $\begin{array}{lllllllll}\text { Superior } & \ldots & \ldots & \ldots & \ldots & \ldots & \ldots & \ldots & \ldots \\
\text { Medio } & \ldots & \ldots & \ldots & \ldots & \ldots & \ldots & \ldots & \ldots \\
\text { Inferior } & \ldots & \ldots & \ldots & \ldots & \ldots & \ldots & \ldots & \ldots\end{array}$ & $\begin{array}{l}+0,83 \\
+0,89 \\
+0,60\end{array}$ & $\begin{array}{l}+0,17 \\
+0,56 \\
+0,30\end{array}$ & $\begin{array}{l}+0,71 \\
+1,00 \\
+0,98\end{array}$ \\
\hline \multicolumn{4}{|l|}{ Grupos de sexo: } \\
\hline 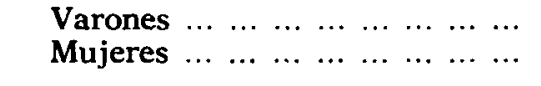 & $\begin{array}{l}+0,67 \\
+0,85\end{array}$ & $\begin{array}{l}+0,33 \\
+0,38\end{array}$ & $\begin{array}{l}+0,96 \\
+0,88\end{array}$ \\
\hline Totales $\ldots \ldots \ldots \ldots$ & $+0,78$ & $+0,36$ & $+0,93$ \\
\hline
\end{tabular}

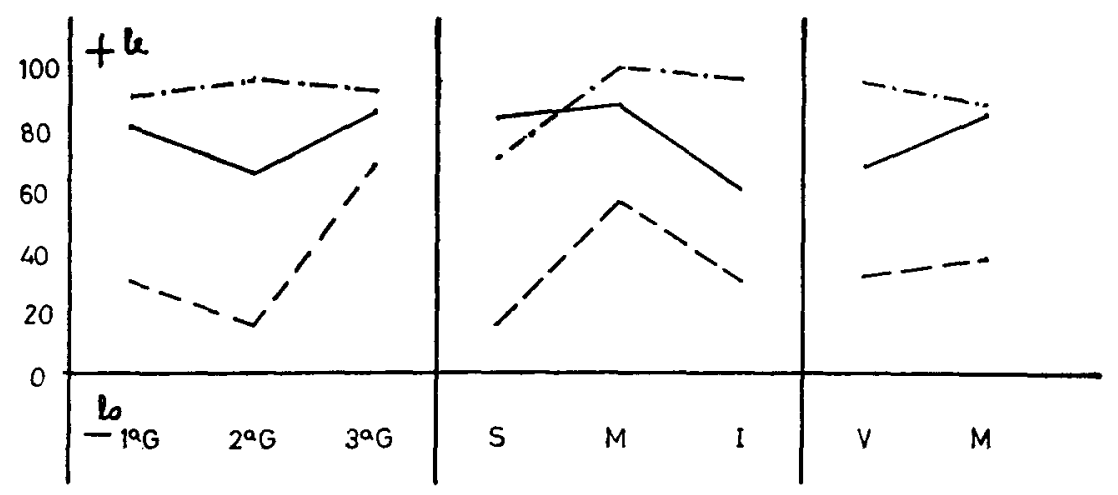

creencia - ; corrección - - - 
en comparación con el leísmo singular desde los inicios, allá por el siglo XIV. Así opina Lapesa:

«De ahí que el leísmo se limite casi al masculino y cunda más en singular que en plural, donde no hay neutro romance» 15.

Nosotros podemos afirmar que en el habla de Burgos no existen tales diferencias entre el leísmo en singular y en plural, ni entre el laísmo de ambos números. El cuadro 3 ofrece los siguientes datos:

\section{CUADRO 3}

Distribución del laísmo y del leismo en singular y en plural por estilos contextuales

\begin{tabular}{|c|c|c|c|c|}
\hline \multirow{2}{*}{$\begin{array}{c}\text { Estilos } \\
\text { contextuales }\end{array}$} & \multicolumn{2}{|c|}{ LEfSMO } & \multicolumn{2}{|c|}{ LAfSMO } \\
\hline & Singular & Plural & Singular & Plural \\
\hline $\begin{array}{ccccc}\text { Lectura } & \ldots & \ldots & \ldots & \ldots\end{array}$ & $\begin{array}{cc}\text { le } & \text { lo } \\
(192) & (8) \\
0,96 & 0,04 \\
\varepsilon= \pm & 0,03\end{array}$ & $\begin{array}{cc}\text { les los } \\
(231) & (19) \\
0,92 & 0,08 \\
\varepsilon= \pm 0,03\end{array}$ & $\begin{array}{cc}\text { le } & \text { la } \\
(76) & (74) \\
0,51 & 0,49 \\
\varepsilon= \pm 0,08\end{array}$ & $\begin{array}{cc}\text { les } & \text { las } \\
(64) & (86) \\
0,43 & 0,57 \\
\varepsilon= \pm 0,08\end{array}$ \\
\hline Respuesta . ... ... ... & $\begin{array}{cc}(44) & (6) \\
0,88 & 0,12 \\
\varepsilon= \pm 0,09\end{array}$ & $\begin{array}{cc}(43) & (7) \\
0,86 & 0,14 \\
\varepsilon= \pm 0,10\end{array}$ & $\begin{array}{cc}(23) & (27) \\
0,46 & 0,54 \\
\varepsilon= \pm 0,14\end{array}$ & $\begin{array}{lr}(19) & (31) \\
0,38 & 0,62 \\
\varepsilon= \pm 0,13\end{array}$ \\
\hline Conversación . ... ... & $\begin{array}{cc}(39) & (4) \\
0,91 & 0,09 \\
\varepsilon= \pm 0,09\end{array}$ & $1^{(35)}(0)$ & $\begin{array}{cc}(7) & (42) \\
0,14 & 0,86 \\
\varepsilon= \pm 0,10\end{array}$ & $\begin{array}{cc}(2) & (11) \\
0,15 & 0,85 \\
\varepsilon= \pm 0,19\end{array}$ \\
\hline $\begin{array}{llll}\cdots & \cdots & \cdots & \text { SaTvLoL }\end{array}$ & $\begin{array}{l}(275)(18) \\
0,94 \quad 0,06 \\
\varepsilon= \pm 0,03\end{array}$ & $\begin{array}{l}(309)(26) \\
0,92 \quad 0,08 \\
\varepsilon= \pm 0,03\end{array}$ & $\begin{array}{l}(106)(143) \\
0,43 \quad 0,57 \\
\varepsilon= \pm 0,06\end{array}$ & $\begin{array}{l}(85)(128) \\
0,40 \quad 0,60 \\
\varepsilon= \pm 0,07\end{array}$ \\
\hline
\end{tabular}

De los datos inducimos que no aparecen diferencias apreciables entre las frecuencias del leísmo en singular y en plural: le ofrece un 94 por 100 de ocurrencias con un $\varepsilon= \pm 3$ por 100 , y les un 92 por 100 con $\varepsilon= \pm 3$ por 100. Lo presenta un 6 por 100 de ocurren-

15 LAPESA, R., op. cit. (1968), pág. 541. 
cias y $\operatorname{los}$ un 8 por 100 con $\varepsilon= \pm 3$ por $100^{16}$. Como se ve, las diferencias son despreciables. Pero es que, además, tampoco parece que las diferencias singular-plural tengan valores sociolingüísticos, pues los estilos contextuales arrojan proporciones prácticamente similares.

En resumen, los hablantes leístas lo son tanto para el singular como para el plural, y lo mismo sucede con los laistas. No tiene mucho sentido, a nuestro juicio, defender, tolerar o admitir el leísmo singular para el sistema funcional o etimológico. En caso contrario lo que se consigue es generar mayor inseguridad y abrir una brecha profunda en el sistema porque la presión analógica del morfema número incitará a los hablantes a generalizar el leísmo al plural.

\section{RASGOS SOCIOLINGÜISTICOS DEL LEfSMO Y LAfSMO}

4.0. Tanto el leísmo como el laísmo son considerados desviaciones propias de Castilla y León. A partir de estos focos se han extendido considerablemente por otros lugares como Castilla la Nueva, zonas de Salamanca que lindan con Extremadura, Santander. Son zonas donde la lucha entre el sistema funcional y el de género aún sigue abierta.

\subsection{El leismo de persona en Burgos}

Los datos del cuadro 4 son elocuentes. En el sistema de habla de Burgos, $l e(s)$ aparece con una frecuencia de 0,93 con un $\varepsilon= \pm 0,02$, mientras que $l o(s)$ da una frecuencia de $0,07 \varepsilon= \pm 0,02$. El leísmo de persona es prácticamente total ${ }^{17}$.

16 Para medir la consistencia de los datos hemos utilizado tres estadísticos. La fiabilidad de la proporción, $\varepsilon$, que nos indica los márgenes entre los que la proporción puede variar en el 95 por 100 de los casos. La diferencia de proporciones, cuando es mayor o igual a 1,96, nos indica que en la diferencia entre las dos muestras no incide el azar más que en el 5 por 100 de las veces que se repitiera el experimento en idénticas condiciones. El test de Pearson o $\chi^{2}$ señala si las distintas muestras pertenecen o no a la misma población. En el caso de que el $\chi^{2}$ de la muestra sea mayor o igual que el muestreo, se supone que las muestras son de distinta población, con un margen de error igual o inferior al 5 por 100 .

17 Entendemos por Sistema de habla o nivel de vigencia la suma total de variantes que se producen en la realización de una variable en todos los estilos contextuales. En este estudio hay un pequeño sesgo - no cuantificado- hacia el estilo más formal. Para que el lector tenga una idea de la diferencia hemos 


\section{CUADRO 4}

Distribución de las variantes le(s) y lo(s), objeto directo masculino de persona, por generaciones $y$ por estilos contextuales

\begin{tabular}{|c|c|c|c|c|}
\hline \multirow{2}{*}{$\begin{array}{c}\text { Estilos } \\
\text { contextuales }\end{array}$} & \multicolumn{4}{|c|}{ GENERACIONES } \\
\hline & $1.0^{\circ} G$. & $2 .{ }^{*} G$. & 3. $G$. & TOtal \\
\hline & le(s) lo(s) & le(s) lo(s) & le(s) lo(s) & le(s) lo(s) \\
\hline $\begin{array}{ccccc}\text { Lectura } & \ldots & \ldots & \ldots & \ldots\end{array}$ & $\begin{array}{ll}(164) & (16) \\
0,91 & 0,09\end{array}$ & $\begin{array}{ll}(149) & (5) \\
0,97 & 0,03\end{array}$ & $\begin{array}{lr}(110) & (7) \\
0,94 & 0,06\end{array}$ & $\begin{array}{ll}(423) & (27) \\
0,94 & 0,06\end{array}$ \\
\hline Respuesta . ... ... & $\begin{array}{ll}(36) & (4) \\
0,90 & 0,10\end{array}$ & $\begin{array}{ll}(29) & (5) \\
0,85 & 0,15\end{array}$ & $\begin{array}{cc}(22) & (4) \\
0,85 & 0,15\end{array}$ & $\begin{array}{cc}(87) & (13) \\
0,87 & 0,13\end{array}$ \\
\hline Conversación & $\begin{array}{rr}(31) & (1) \\
0,97 & 0,03\end{array}$ & $\begin{array}{cc}(24) & (1) \\
0,96 & 0,04\end{array}$ & $\begin{array}{ll}(19) & (2) \\
0,90 & 0,10\end{array}$ & $\begin{array}{cc}(74) & (4) \\
0,95 & 0,05\end{array}$ \\
\hline Totales $\ldots$ & $\begin{array}{l}(231) \\
0,92 \quad 0,08 \\
\varepsilon= \pm 0,03\end{array}$ & $\begin{array}{l}(202) \\
0,95 \quad 0,05 \\
\varepsilon= \pm 0,03\end{array}$ & $\begin{array}{l}(151)(13) \\
0,920,08 \\
\varepsilon= \pm 0,04\end{array}$ & $\begin{array}{ll}(584) & (44) \\
0,93 \quad 0,07 \\
\varepsilon= \pm 0,02\end{array}$ \\
\hline $\begin{array}{c}\text { Media de proporcio } \\
\text { nes } \ldots \ldots \\
\ldots\end{array}$ & $0,93 \quad 0,07$ & $0,93 \quad 0,07$ & $0,90 \quad 0,10$ & $0,920,08$ \\
\hline
\end{tabular}

Los estilos contextuales apenas ofrecen diferencias entre ellos, por lo que suponemos que los caracteres sociolingüísticos serán mínimos al no existir presión desde ningún nivel de la consciencia de habla. La frecuencia de $l e(s)$ en el estilo «Lectura», el más formal, es prácticamente igual a la del estilo «Conservación», el más informal: 0,94-0,95. El estilo aRespuesta», a medio camino entre la formalidad plena y la informalidad, ofrece diferencias debidas al azar $\mathrm{Dp}=1,92$.

Por generaciones no se aprecian diferencias en el comportamiento ante $l e(s)$ y $l o(s)$. El leísmo está extendido por igual y por todas las generaciones; no parece haber cambios en tiempo aparente.

Los niveles socioculturales están representados en el cuadro 5.

optado por incluir en los datos la media de las proporciones, procedimiento no muy riguroso estadísticamente, pero útil para nuestro propósito. 


\section{CUADRO 5}

Distribución de las variantes le(s) y $l o(s)$ objeto directo masculino de persona, por niveles socioculturales $y$ estilos contextuales

\begin{tabular}{|c|c|c|c|c|}
\hline \multirow{2}{*}{$\begin{array}{c}\text { Estilos } \\
\text { contextuales }\end{array}$} & \multicolumn{4}{|c|}{ NIVELES SOCIOCULTURALES } \\
\hline & Superior & Medio & Inferior & TOTAL \\
\hline $\begin{array}{lllll}\text { Lectura } & \ldots & \ldots & \ldots & \ldots\end{array}$ & $\begin{array}{rr}l e(s) & l o(s) \\
(91) & (17) \\
0,84 & 0,16\end{array}$ & $\begin{array}{l}\text { le(s) lo(s) } \\
(162)(-) \\
1\end{array}$ & $\begin{array}{l}l e(s) l o(s) \\
(170)(10) \\
0,940,06\end{array}$ & $\begin{array}{ll}\text { le(s) } & \text { lo(s) } \\
(423) & (27) \\
0,94 & 0,06\end{array}$ \\
\hline Respuesta . ... ... ... & $\begin{array}{ll}(19) & (5) \\
0,79 & 0,21\end{array}$ & $\begin{array}{rr}(34) & (2) \\
0,94 & 0,06\end{array}$ & $\begin{array}{cc}(34) & (6) \\
0,85 & 0,15\end{array}$ & $\begin{array}{rr}(87) & (13) \\
0,87 & 0,13\end{array}$ \\
\hline Conversación . ... ... & ${ }_{1}^{(17)} \underset{0}{(-)}$ & $\begin{array}{ll}(23) & (1) \\
0,96 & 0,04\end{array}$ & $\begin{array}{cc}(34) & (3) \\
0,92 & 0,08\end{array}$ & $\begin{array}{cc}(74) & (4) \\
0,95 & 0,05\end{array}$ \\
\hline Totales $\ldots \ldots \ldots$ & $\begin{array}{ll}(127) & (22) \\
0,85 & 0,15 \\
\varepsilon \pm=0,06\end{array}$ & $\begin{array}{cc}(219) & (3) \\
0,99 & 0,01 \\
\varepsilon= \pm 0,02\end{array}$ & $\begin{array}{l}(238)(19) \\
0,930,07 \\
\varepsilon= \pm 0,03\end{array}$ & $\begin{array}{ll}(584) & (44) \\
0,93 & 0,07\end{array}$ \\
\hline $\begin{array}{c}\text { Media de proporcio- } \\
\text { nes } \begin{array}{llllll} & \ldots & \ldots & \ldots & \ldots & \ldots\end{array}\end{array}$ & $0,90 \quad 0,10$ & $0,97 \quad 0,03$ & $0,90 \quad 0,10$ & $0,920,08$ \\
\hline
\end{tabular}

Los datos del cuadro 5 nos demuestran que lo prestigioso no es $l o(s)$, sino $l e(s)$, que es el uso más generalizado. Es probable que sea su misma extensión la que proporcione a $l e(s)$ el prestigio necesario.

Grupos de sexo: El cuadro 6 nos revela que el comportamiento de varones y mujeres es, en general, idéntico: predomina el leísmo y las cortas diferencias que existen se deben al azar.

En conclusión: Burgos es plenamente leísta. Los pocos casos de $l o(s)$ no tienen suficiente entidad y, además, no son imitados por el grupo sociocultural medio; carecen de prestigio ${ }^{18}$. Por otra parte,

18 Hemos podido comprobar que los pocos ejemplos de uso de lo (s) como objeto directo masculino que ofrece el habla conversacional burgalesa tiene como antecedentes, principalmente, palabras como niño, hijo, crio (de criatura):

aLo cogí así de los brazos y.... (informante 35).

¿Un hijo lo cria su madre (informante 7 ).

o se refiere a alguien que ya no está en el mundo de los vivos, quizá una fosilización:

a... al difunto Manero, que Dios $l o$ tenga en gloria» (informante 43). 


\section{CUADRO 6}

Distribución de las variantes le(s) y lo(s) objeto directo masculino por grupos de sexto y estilos contextuales

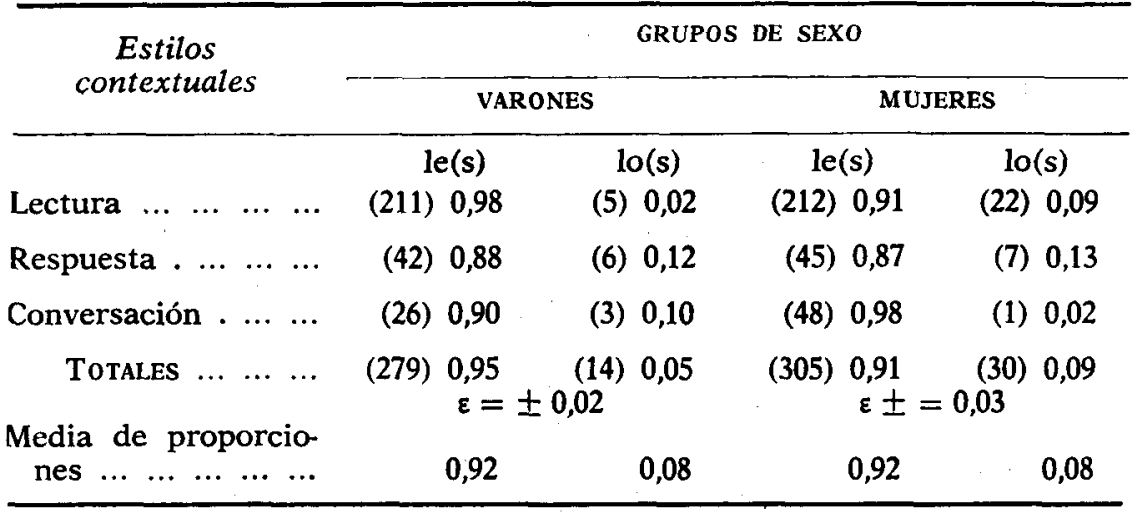

no hay tendencia al cambio en tiempo aparente: el leísmo es constante en todas las generaciones. Estamos ante un indicador sociolinguístico - según la terminología de Labov- sin apenas estratificación.

\subsection{El laismo de persona en Burgos}

En el sistema de habla de Burgos se aprecia un ligero predominio del laísmo. Las proporciones son 0,41 con $\varepsilon= \pm 0,04$ para $l e(s)$ objeto indirecto femenino y 0,59 con $\varepsilon= \pm 0,04$ para $l a(s)$ objeto indirecto femenino. Hay un polimorfismo alternante con predominio laísta de 18 puntos. Conviene tener en cuenta que el sistema de habla o nivel de vigencia —l total de filas y columnas- está desviado hacia los estilos de «Lectura» $y$ «Respuesta», los estilos de mayor formalidad. Pero si tomamos la media de las proporciones nos encontramos con un 34 por 100 de $l e$ frente a un 66 por 100 de laísmo; 31 puntos de diferencia, casi el doble.

$\mathrm{Si}$ aislamos los estilos contextuales comienzan a aparecer diferencias importantes. Mientras que los estilos contextuales de «Lectura» y de «Respuesta» presentan una distribución semejante, en el estilo "Conservación» la presencia de $l a(s)$ sube muchos puntos en relación con la de $l e(s): 0,85$ frente a 0,15 con un $\varepsilon= \pm 0,09$. Tales 
diferencias estilísticas nos hacen pensar que aparecerán diferencias sociolingüísticas importantes. Aislaremos las variables sociales para observar estas posibles diferencias.

- Generaciones:

\section{CUADRO 7}

Distribución de la(s) y le(s) objeto indirecto femenino, por generaciones $y$ estilos contextuales

\begin{tabular}{|c|c|c|c|c|}
\hline \multirow{2}{*}{$\begin{array}{c}\text { Estilos } \\
\text { contextuales }\end{array}$} & \multicolumn{4}{|c|}{ GENERACIONES } \\
\hline & 1.: $G$. & $2 *^{*} G$. & 3. $G$. & TOTAL \\
\hline & le(s) la(s) & $l e(s) l a(s)$ & le(s) la(s) & $\operatorname{le}(\mathrm{s}) \mathrm{la}(\mathrm{s})$ \\
\hline $\begin{array}{ccccc}\text { Lectura } & \ldots & \ldots & \ldots & \ldots\end{array}$ & $\begin{array}{ll}(66) & (53) \\
0,55 & 0,45\end{array}$ & $\begin{array}{ll}(38) & (64) \\
0,37 & 0,63\end{array}$ & $\begin{array}{ll}(36) & (42) \\
0,46 & 0,54\end{array}$ & $\begin{array}{l}(140)(162) \\
0,46 \quad 0,54 \\
\varepsilon= \pm 0,06\end{array}$ \\
\hline Respuesta . ... ... ... & $\begin{array}{ll}(20) & (20) \\
0,50 & 0,50\end{array}$ & $\begin{array}{cc}(13) & (21) \\
0,38 & 0,62\end{array}$ & $\begin{array}{cc}(9) & (17) \\
0,35 & 0,65\end{array}$ & $\begin{array}{lc}(42) & (58) \\
0,42 & 0,58 \\
\varepsilon= \pm 0,10\end{array}$ \\
\hline Conversación . ... ... & $\begin{array}{cc}(2) & (9) \\
0,18 & 0,82\end{array}$ & $\begin{array}{cc}(4) & (14) \\
0,22 & 0,78\end{array}$ & $\begin{array}{rr}(3) & (30) \\
0,09 & 0,91\end{array}$ & $\begin{array}{cc}(9) & (53) \\
0,15 & 0,85 \\
\varepsilon=+0,09\end{array}$ \\
\hline Totales $\ldots \ldots \ldots$ & $\begin{array}{ll}(88) & (82) \\
0,52 & 0,48 \\
\varepsilon= \pm 0,08\end{array}$ & $\begin{array}{ll}(55) & (99) \\
0,36 & 0,64 \\
\varepsilon= \pm 0,08\end{array}$ & $\begin{array}{ll}(48) & (89) \\
0,35 & 0,65 \\
\varepsilon= \pm 0,08\end{array}$ & $\begin{array}{l}(191) \\
0,41 \quad 0,59 \\
\varepsilon= \pm 0,04\end{array}$ \\
\hline $\begin{array}{c}\text { Media de proporcio } \\
\text { nes } \ldots \ldots \\
\ldots\end{array}$ & $0,41 \quad 0,59$ & $0,32 \quad 0,68$ & $0,30 \quad 0,70$ & $0,34 \quad 0,66$ \\
\hline
\end{tabular}

El hecho que más llama la atención al observar el cuadro 7 es que en los estilos contextuales más formales es la primera generación la que menos casos de laísmo ofrece. Son cortas las diferencias; lo cual nos hace pensar que el influjo escolar puede ser el determinante, pues en la conversación la realización laísta adquiere fuerza en todas las generaciones. 


\section{CUADRO 8}

Distribución de la(s) y le(s) objeto indirecto femenino, por niveles socioculturales y estilos contextuales

\begin{tabular}{|c|c|c|c|}
\hline \multirow{2}{*}{$\begin{array}{c}\text { Estilos } \\
\text { contextuales }\end{array}$} & \multicolumn{3}{|c|}{ NIVELES SOCIOCULTURALES } \\
\hline & Superior & Medio & Inferior \\
\hline $\begin{array}{lllll}\text { Lectura } & \ldots & \ldots & \ldots & \ldots\end{array}$ & $\begin{array}{cc}\mathrm{le}(\mathrm{s}) & \mathrm{la}(\mathrm{s}) \\
(51) & (21) \\
0,71 & 0,29 \\
\varepsilon= \pm & 0,11\end{array}$ & $\begin{array}{cc}\operatorname{le}(\mathrm{s}) & \mathrm{la}(\mathrm{s}) \\
(45) & (63) \\
0,42 & 0,58 \\
\varepsilon= \pm & 0,09\end{array}$ & $\begin{array}{cc}\operatorname{le}(\mathrm{s}) & \mathrm{la}(\mathrm{s}) \\
(44) & (76) \\
0,37 & 0,63 \\
\varepsilon= \pm 0,09\end{array}$ \\
\hline Respuesta . ... ... ... & $\begin{array}{cc}(15) & (9) \\
0,63 & 0,38 \\
\varepsilon= \pm 0,19\end{array}$ & $\begin{array}{cc}(14) & (22) \\
0,39 & 0,61 \\
\varepsilon= \pm 0,16\end{array}$ & $\begin{array}{lr}(13) & (27) \\
0,33 & 0,68 \\
\varepsilon= \pm 0,15\end{array}$ \\
\hline Conversación . ... ... & $\begin{array}{l}(3) \\
0,25 \\
\varepsilon= \pm 0,25\end{array}$ & $\begin{array}{cc}(2) & (16) \\
0,11 & 0,89 \\
\varepsilon= \pm & 0,14\end{array}$ & $\begin{array}{cc}(4) & (28) \\
0,13 & 0,87 \\
\varepsilon= \pm 0,12\end{array}$ \\
\hline TOtales $\ldots \ldots \ldots$ & $\begin{array}{cc}(69) & (39) \\
0,64 & 0,36 \\
\varepsilon= \pm 0,09\end{array}$ & $\begin{array}{cr}(61) & (101) \\
0,38 & 0,62 \\
\varepsilon= \pm & 0,07\end{array}$ & $\begin{array}{cc}(61) & (131) \\
0,32 & 0,68 \\
\varepsilon= \pm 0,07\end{array}$ \\
\hline $\begin{array}{llllll}\text { nes } & \ldots & \ldots & \ldots & \ldots & \ldots\end{array}$ & 0,53 & 0,31 & 0,28 \\
\hline
\end{tabular}

Si nos fijamos en los estilos contextuales observamos que el laísmo aumenta por igual en todos los grupos según descendemos en el grado de consciencia de habla. El $\chi^{2}$ de esta distribución es de $1,03<9,58$ que es nuestro $\chi^{2}$. Esto demuestra que no hay diferencias de comportamiento entre los diferentes grupos; parece que los informantes tienen conciencia, cada uno en su nivel, del posible valor sociolingüístico de la alternancia.

Pues bien, observamos una curva ascendente en el uso de la(s) que distingue al grupo sociocultural superior del medio $\mathrm{y}$ del inferior (ver gráfico 3). El grupo medio y el inferior se comportan, respecto al laísmo, de forma similar, con sólo seis puntos de diferencia que se deben al azar. Sin embargo, si comparamos las proporciones 
del grupo superior con las del medio, la diferencia es de 26 puntos y no se debe al azar; Dp: $0,26 / 0,06=4,47>1,96{ }^{19}$.

\section{GRAFICO 3}

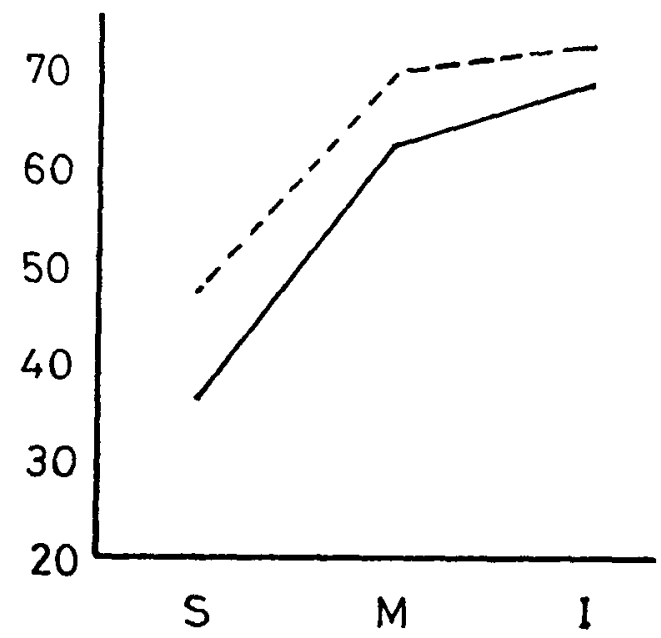

Laismo por niveles socioculturales en el total de los estilos contextuales

- - Media de proporciones.

Total de valores absolutos en porcentaje.

El gráfico 3 nos señala que el laísmo es menos utilizado por hablantes del nivel sociocultural superior y que asciende vertiginosamente en los demás grupos socioculturales. Parece que es amás cultow el uso de $l e(s)$ objeto indirecto femenino, pero el prestigio no se manifiesta en toda su potencia, pues no tiene fuerza suficiente para hacer que el grupo sociocultural medio imite el comportamiento del superior. Esto quiere decir que el laísmo no es un $\mathrm{f} \theta$ nómeno estigmatizado negativamente; por esta razón ofrece una fre-

19 Obsérvese que el perfil de la curva es muy similar a la que ofrece KuBiN Flora, op. cit., pág. 300, aunque nuestros porcentajes son algo más bajos. Conviene recordar que la autora da datos sólo de estilo informal (conversación) de varones y que muchos hablantes pertenecen a zonas rurales. 
cuencia de aparición más alta que le(s) en el estilo de habla menos formal $\mathbf{y}$ en todos los grupos socioculturales.

- Grupos de sexo: El laísmo aumenta por igual y muy equilibradamente en ambos grupos al descender en el grado de consciencia de habla. En el total de los datos no se aprecian diferencias.

\section{Conclusión}

5.1. Los caracteres sociolingüísticos del laísmo y del leísmo de persona en la ciudad de Burgos son muy similares por variables sociales; pero mientras el leísmo es un indicador sociolinguiístico, el laísmo es un marcador sociolingüístico, según la terminología de Labov. La diferencia esencial está en que la generalización del leísmo se da incluso en el habla más formal, lo que supone una aceptación completa del fenómeno por parte de todos los hablantes. El laísmo, por el contrario, muestra grandes diferencias estilísticas en todas las variables sociales. Hay predominio de laísmo en el estilo informal pero, en los estilos formales, es patente un polimorfismo alternante equilibrado aunque con importantes desviaciones a favor del laísmo. Esto produce una menor frecuencia general de laísmo en el nivel sociocultural superior pero sin llegar a una estigmatización negativa del fenómeno, ya que ni $l e(s)$ es imitado por el grupo medio, ni el superior intenta evitar el laísmo en el estilo informal. Hay leísmo casi total y laísmo en los estilos informales. El leísmo es prestigioso y el laísmo no está estigmatizado negativamente.

5.2. El sistema burgalés es un sistema basado, predominantemente, en la distinción por el género, en cuanto a personas se refiere. Existe una constricción importante en el objeto indirecto femenino cuando el hablante es consciente de su habla, y esta constricción impide que se generalice la oposición basada en el género.

5.3. Por último, daremos unos datos sobre el loísmo. El loísmo objeto indirecto masculino es prácticamente inexistente en el habla de Burgos. En el estilo "Lectura" aparecen cinco casos - siempre en plural- de un total de 100 posibles ocurrencias; en el estilo "Respuesta" aparecen tres casos, dos en plural y uno en singular; en el estilo «Conversación» sólo aparecen tres casos sobre un total de 138 ocurrencias y siempre en plural. Además, los pocos casos que aparecen no ofrecen diferencias por variables externas. 